\title{
AVALIAÇÃO DE ICP OES COM CONFIGURAÇÃO AXIAL OU RADIAL PARA DETERMINAÇÃO DE IODO EM SAL DE COZINHA
}

\author{
Adriana A. Oliveira", Joaquim A. Nóbrega* e Edenir R. Pereira-Filho \\ Departamento de Química, Universidade Federal de São Carlos, CP 676, 13560-970 São Carlos - SP, Brasil \\ Lilian C. Trevizan ${ }^{\# \#}$ \\ Centro de Energia Nuclear na Agricultura, Universidade de São Paulo, CP 96, 13400-970 Piracicaba - SP, Brasil
}

Recebido em 8/5/11; aceito em 23/2/12; publicado na web em 15/6/12

\begin{abstract}
EVALUATION OF ICP OES WITH AXIAL OR RADIAL VIEWS FOR DETERMINATION OF IODINE IN TABLE SALT. The performance of inductively-coupled plasma optical emission spectrometers with axial and radial views for determination of iodine in table salt was evaluated. Interference and memory effects in nitric acid and water-soluble tertiary amines (CFA-C) media were studied. Based on a factorial experiment, one optimum instrument operational condition for axial configuration, and two optima conditions for radial configuration was established. The ICP OES with axial view was 5-fold more sensitive than the radial view. Both matrix matching and standard addition methods were used for iodine quantification and for most samples, both strategies of calibration led to similar results. Recoveries ranged from 104 to $114 \%$.
\end{abstract}

Keywords: iodine; ICP OES; table salt.

\section{INTRODUÇÃO}

O iodo é essencial para a formação de tirosina $\left(\mathrm{T}_{4}\right)$ e de tri-iodotironina $\left(\mathrm{T}_{3}\right)$, dois hormônios da tireoide, importantes para o controle de diversas funções do corpo humano, tais como metabolismo celular, temperatura corporal, reprodução e crescimento.

A carência de iodo pode causar mau funcionamento (hipotireoidismo) ou doenças da glândula tireoide (bócio), cretinismo, retardo mental, aumento de mortalidade perinatal e desenvolvimento de anomalias. ${ }^{1}$ Entretanto, o consumo exagerado pode levar ao aumento das doenças autoimunes da tireoide e ao hipertireoidismo. ${ }^{2}$

Quantidades elevadas de iodo são encontradas nos oceanos e em alimentos de origem marinha. Exceto para populações que vivem em regiões litorâneas, os alimentos marinhos são consumidos em pequenas quantidades, sendo assim, ovos e leite são as principais fontes de iodo. No entanto, o teor de iodo encontrado nesses alimentos ainda não é suficiente para suprir as necessidades diárias recomendadas pela OMS (Organização Mundial de Saúde). Uma das maneiras de supri-las na alimentação da população é a adição desse elemento aos alimentos. No Brasil, em março de 2003, a Agência Nacional de Vigilância Sanitária (ANVISA) estabeleceu que o sal destinado ao consumo humano deve conter uma concentração de iodo igual ou superior a $20 \mathrm{mg} \mathrm{kg}^{-1}$ até o limite máximo de $60 \mathrm{mg} \mathrm{kg}^{-1}$. $^{3}$

Métodos espectrofotométricos clássicos para determinação de iodo envolvem o complexo colorido formado entre iodo e $\operatorname{amido}^{4} \mathrm{e}$, também, reações catalíticas nas quais o iodeto age como catalisador na reação redox entre Ce(IV) (amarelo) e As(III) formando os íons incolores $\mathrm{Ce}(\mathrm{III})$ e As(V). Essa reação catalítica é conhecida como reação de Sandell-Kolthoff e por muitos anos representou o estado da arte para a determinação de iodo em materiais biológicos. ${ }^{5,6}$

Além de procedimentos espectrofotométricos bem estabelecidos, ${ }^{4}$ algumas técnicas têm sido aplicadas para a determinação de iodo em

*e-mail: djan@ufscar.br

"Endereço atual: Corn Products, Mogi Guaçu - SP

\#"Endereço atual: Divisão de Controle Sanitário e Ambiental, Unidade de Negócio do Médio Tietê, Cia. de Saneamento Básico do Estado de São Paulo, Botucatu - SP amostras variadas, incluindo espectrometria de absorção atômica com chama (FAAS, Flame Atomic Absorption Spectrometry), ${ }^{7}$ análise por ativação neutrônica (NAA, Neutron Activation Analysis), ${ }^{8}$ fluorescência de raios-X com reflexão total (TXRF, Total reflection $X$-ray Fluorescence), ${ }^{9}$ espectrometria de massa acoplada ao plasma indutivo (ICP-MS, Inductively Coupled Plasma Mass Spectrometry) e espectrometria de emissão óptica com plasma acoplado indutivamente (ICP OES, ICP Optical Emission Spectrometry).

O desenvolvimento de métodos para a determinação de iodo em alimentos e no sal de cozinha, aliado à robustez do ICP OES é importante para análises de rotina. Entretanto, poucos trabalhos na literatura relatam a determinação de não metais por ICP OES.

Medidas na região do vácuo ultravioleta (VUV), que corresponde a comprimentos de onda abaixo de $190 \mathrm{~nm}$, possibilitam a determinação de não metais, isto é, $\mathrm{C}, \mathrm{Cl}, \mathrm{Br}$, I, P e S, com sensibilidade adequada. ${ }^{10-14}$ Essas medidas apresentam algumas dificuldades, principalmente para halogênios, devido à elevada energia de ionização dos mesmos (por exemplo, I 10,45 eV) comparada à maioria dos elementos. Consequentemente, a ionização desses elementos, no plasma de argônio, será incompleta, levando à formação de um plasma relativamente pobre em átomos excitados e, portanto, altos limites de detecção (LOD). No caso do I, aproximadamente $29 \%$ dos átomos estarão na forma ionizada. ${ }^{15}$ Outros problemas são a absorção da radiação emitida na região VUV por componentes do ar, como oxigênio e vapor de água, e também a disponibilidade de um detector sensível nessa região. Entretanto, o desenvolvimento de sistemas ópticos purgados com gases, como $\mathrm{Ar}$ ou $\mathrm{N}_{2}$, possibilita a remoção de espécies que absorvem a radiação e viabilizam a medida. Interfaces adequadas para a remoção da região mais fria do plasma possibilitam uma diminuição significativa de interferências no sistema axial e a determinação na região do VUV do espectro eletromagnético.

Outro aspecto crítico na determinação de iodo é o preparo de amostra, pois pode haver perda desse elemento por volatilização, principalmente quando é empregada a decomposição ácida. Nesse caso, é comum a formação de compostos voláteis de iodo, como $\mathrm{I}_{2}$ e HI, que além de provocar perda do analito também causam efeito de memória quando a solução é introduzida no atomizador por nebulização pneumática. Uma alternativa para tal problema é a 
aplicação de meios alcalinos, como hidróxido de sódio, hidróxido de tetrametilamônio (TMAH, tetramethylammonium hydroxide) ou soluções de aminas terciárias solúveis em água (CFA-C, water-soluble tertiary amines solution). ${ }^{6}$

O objetivo deste trabalho consistiu na avaliação do comportamento do iodo em ICP OES com configuração axial ou radial. Além disso, foi avaliado o desempenho do ICP OES com configuração axial para a determinação de iodo em amostras de sal de cozinha. A presença de elevadas concentrações de sódio causa um elevado sinal de fundo, potencializa interferências espectrais, além de causar dificuldades com o ICP OES com configuração axial e pode reduzir drasticamente a vida útil da tocha de quartzo. Ambos os efeitos dificultam a determinação de iodo em sal de cozinha por emissão atômica em plasma.

\section{PARTE EXPERIMENTAL}

\section{Instrumentação}

Utilizou-se um ICP OES com configuração axial (Vista AX, Varian, Mulgrave, Victoria, Austrália), equipado com interface end-on (isto é, fluxo de Ar introduzido frontalmente ao plasma) para remoção da zona fria do plasma, detector de estado sólido CCD (charge-coupled device) refrigerado a $-35{ }^{\circ} \mathrm{C}$ para possibilitar medidas de 167 a $785 \mathrm{~nm}$ e sistema pré-óptico purgado com vazão de $\operatorname{Ar}$ de $3 \mathrm{~L} \mathrm{~min}{ }^{-1}$. Também se usou um ICP OES com configuração radial (Vista RD, Varian), equipado com detector de estado sólido CCD refrigerado a $-35{ }^{\circ} \mathrm{C}$ e sistema pré-óptico purgado com vazão de $\mathrm{Ar}$ de $0,2 \mathrm{~L} \mathrm{~min}^{-1}$. Os parâmetros instrumentais e os comprimentos de onda utilizados na avaliação de iodo encontram-se na Tabela 1.

Tabela 1. Condições operacionais do ICP OES com configuração axial e com configuração radial para a avaliação da determinação de iodo

\begin{tabular}{lcc}
\hline Parâmetros operacionais & Radial & Axial \\
\hline Radiofrequência do gerador (MHz) & 40 & 40 \\
Potência aplicada (kW) & $0,7-1,4$ & $0,7-1,4$ \\
Vazão do gás do plasma $\left(\mathrm{L} \mathrm{min}^{-1}\right)$ & 15 & 15 \\
Vazão do gás auxiliar $\left(\mathrm{L} \mathrm{min}^{-1}\right)$ & 1,5 & 1,5 \\
Vazão do gás de nebulização $\left(\mathrm{L} \mathrm{min}^{-1}\right)$ & $0,5-1,4$ & $0,5-1,4$ \\
Vazão de introdução de amostra & $0,5-4,5$ & $0,5-4,5$ \\
(mL min $\left.{ }^{-1}\right)$ & $4-20$ \\
Altura de observação (mm) & 1,4 & - \\
Diâmetro interno do tubo central (mm) & Sturman-Masters & Sturman-Masters \\
Câmara de nebulização & Ranhura em V & Ranhura em V \\
Nebulizador & (I) $178,215^{*}$ \\
Comprimentos de onda de iodo (nm) & (I) 179,847 \\
& (I) 182,977 \\
& (I) 184,385 \\
& (I) 206,165 \\
\hline
\end{tabular}

*I: linha atômica.

\section{Reagentes, soluções e amostras}

Todos os reagentes empregados foram de grau analítico e as soluções foram preparadas utilizando água destilada-desionizada purificada em sistema Milli-Q ${ }^{\circledast}$ (Millipore, Bedford, MA, EUA, resistividade $18,2 \mathrm{M} \Omega \mathrm{cm}$ ).

A solução analítica de referência foi preparada a partir de HI concentrado (Vetec Química Fina Ltda, RJ, Brasil) em meio de CFA-C (mistura de aminas terciárias solúveis em água, composta por trietanolamina e trietilenoamina - Spectrasol, Warwick, NY, EUA) $10 \% \mathrm{v} \mathrm{v}^{-1}, \mathrm{pH} 8$ e alguns testes também foram realizados em meio de $\mathrm{HNO}_{3}$ (Merck) $1 \% \mathrm{v} \mathrm{v}^{-1}$.

As amostras de sal de cozinha foram adquiridas no comércio local da cidade de São Carlos-SP, sendo que as marcas foram selecionadas aleatoriamente.

\section{Métodos}

Avaliação do efeito de memória

$\mathrm{Na}$ avaliação da ocorrência de efeito de memória utilizou-se solução de KI como padrão de iodo (100 mg L L $)$ em meio de $\mathrm{HNO}_{3}$ $1 \% \mathrm{v} \mathrm{v}^{-1}$ e de CFA-C $10 \% \mathrm{v} \mathrm{v}^{-1} \mathrm{pH} 8$. Monitorou-se a intensidade de emissão de iodo em função do tempo de medidas nesses meios.

\section{Avaliação do desempenho das configurações axial e radial}

Para a avaliação das condições operacionais de ICP OES com configurações axial e radial, na determinação de iodo, foram realizados dois planejamentos fatoriais. No caso do ICP OES com configuração axial, três variáveis foram estudadas: vazão do gás de nebulização, potência aplicada de radiofrequência e vazão de introdução da amostra. Já para a configuração radial, a variável vazão de introdução de amostra foi substituída pela variável altura de observação. Dois planejamentos $2^{3}+$ Ponto Central + Estrela foram efetuados, totalizando 17 experimentos cada, conforme descrito na Tabela 1S, material suplementar. ${ }^{17}$ Todos os experimentos foram realizados em 5 níveis, utilizando-se solução de HI como padrão de iodo $(100 \mathrm{mg}$ $\mathrm{L}^{-1}$ ) em meio de CFA-C $10 \% \mathrm{v} \mathrm{v}^{-1}$ e $\mathrm{pH} 8$. Além disso, o ponto central foi efetuado em triplicata para o cálculo dos erros dos coeficientes de regressão. A Tabela 1S, material suplementar, mostra também os níveis avaliados para cada variável. Esses valores tiveram como base os limites operacionais típicos dos equipamentos. ${ }^{18}$

Os cálculos dos planejamentos fatoriais foram realizados no programa Microsoft Excel (versão 2003) e o programa Surfer 5.0 foi utilizado para a obtenção dos gráficos de contorno, após a realização de todos os experimentos propostos nos planejamentos experimentais.

A avaliação das linhas de emissão de iodo em equipamentos com configurações radial ou axial foi efetuada por medidas da razão sinal analítico/sinal de fundo (SBR - signal-to-background ratio), da concentração equivalente ao sinal de fundo (BEC - background equivalent concentration) e da determinação do limite de detecção (LOD). ${ }^{19}$

Recomenda-se utilizar o conceito de BEC, definido como sendo a concentração do analito que produz sinal equivalente à intensidade de emissão do fundo na linha medida, para o cálculo de LOD em ICP OES. Esse parâmetro possibilita visualizar, principalmente para meios complexos, qualquer mudança no espectro de fundo devido a alterações nas condições operacionais em comparação ao LOD calculado da maneira convencional. ${ }^{20,21}$

Os sinais para as soluções de $\mathrm{HI}$ como padrão de iodo $\left(100 \mathrm{mg} \mathrm{L}^{-1}\right)$ em meio de CFA-C foram medidos em triplicata. Já para as soluções de branco no meio CFA-C $10 \% \mathrm{v} \mathrm{v}^{-1} \mathrm{em} \mathrm{pH} 8$ foram registrados 10 triplicatas. O corretor de fundo foi desabilitado pelo programa de controle do equipamento durante o procedimento e obtiveram-se os valores das intensidades das linhas de emissão de iodo.

Avaliou-se também a faixa linear de calibração para as duas configurações. Na configuração axial, a faixa de concentração estudada foi de 0,3 a $10000 \mathrm{mg} \mathrm{L}^{-1}$ e na configuração radial, a faixa foi de 1,8 a $10000 \mathrm{mg} \mathrm{L}^{-1}$ de iodo. As soluções analisadas foram preparadas em meio de CFA-C $10 \% \mathrm{v} \mathrm{v}^{-1}$ em $\mathrm{pH} 8$.

\section{Avaliação de interferentes}

Para a avaliação de interferentes, adicionaram-se os sais $\mathrm{NaCl}$ e $\mathrm{KCl}$ em soluções de HI, como padrão de iodo (100 mg L-1), em meio 
de CFA-C $10 \% \mathrm{v} \mathrm{v}^{-1}$ em $\mathrm{pH} 8$, para verificar os eventuais efeitos dos cátions $\mathrm{Na}^{+}$ou $\mathrm{K}^{+}$e a mistura $\mathrm{Na}^{+}$e $\mathrm{K}^{+}$na determinação de iodo. As concentrações estudadas dos interferentes foram 100, 250, 500, 750, $1000,2000,4000,6000,8000$ e $10000 \mathrm{mg} \mathrm{L}^{-1}$. Para a mistura contendo $\mathrm{Na}^{+}$e K $\mathrm{K}^{+}$, a concentração de cada cátion encontrava-se na proporção $1: 1 \mathrm{~m} \mathrm{~m}^{-1}$ em relação à concentração final estudada.

\section{Determinação de iodo em amostras de sal de cozinha}

Foram analisadas 9 amostras de sal de cozinha iodado, dentre elas 3 sais comuns, 2 sais com menor teor de $\mathrm{Na}$ (denominados comercialmente como light) e 4 sais marinhos.

O preparo da solução de sal de cozinha iodado foi baseado na quantidade mínima de iodo a ser adicionada, estipulada pela ANVISA, ou seja, $20 \mathrm{mg} \mathrm{kg}^{-1}$ e no LOQ calculado com base no LOD obtido na avaliação das linhas de emissão de iodo $\left(0,1 \mathrm{mg} \mathrm{L}^{-1}\right)$.

Assim, dissolveram-se $500 \mathrm{mg}$ de sal de cozinha iodado em $25 \mathrm{~mL}$ de solução de CFA-C $10 \% \mathrm{v} \mathrm{v}^{-1}$ em pH 8, para que a solução final tivesse concentração próxima ao LOQ, ou seja, $0,3 \mathrm{mg} \mathrm{L}^{-1}$ de iodo, supondo-se que o produto atenda ao estabelecido pela legislação.

Foram preparadas soluções analíticas de calibração em dois meios. Um conjunto de soluções foi preparado em meio de CFA-C $10 \% \mathrm{v} \mathrm{v}^{-1} \mathrm{em} \mathrm{pH} 8+\mathrm{NaCl}\left(8000 \mathrm{mg} \mathrm{L}^{-1} \mathrm{de} \mathrm{Na}^{+}\right)$de grau analítico e outro em meio de CFA-C $10 \% \mathrm{v} \mathrm{v}^{-1} \mathrm{em} \mathrm{pH} 8+\mathrm{NaCl}\left(4000 \mathrm{mg} \mathrm{L}^{-1} \mathrm{de}\right.$ $\left.\mathrm{Na}^{+}\right)+\mathrm{KCl}\left(4000 \mathrm{mg} \mathrm{L}^{-1}\right.$ de $\left.\mathrm{K}^{+}\right)$de grau analítico. As concentrações de iodo nas soluções analíticas de calibração foram 0,$1 ; 0,3 ; 0,5 ; 1$ e $3 \mathrm{mg} \mathrm{L}^{-1}$

Para a quantificação das amostras, também se adotou o método de calibração por adições de padrão. Nesse estudo, foi preparada uma solução $4 \%(\mathrm{~m} / \mathrm{v})$ de sal de cozinha iodado em meio de CFA-C $10 \% \mathrm{v} \mathrm{v}^{-1} \mathrm{em} \mathrm{pH} \mathrm{8,} \mathrm{retirou-se} \mathrm{uma} \mathrm{alíquota} \mathrm{de} 25 \mathrm{~mL}$ dessa solução e transferiu-se para um balão volumétrico de $50 \mathrm{~mL}$. Em cada balão volumétrico adicionaram-se concentrações crescentes de iodo $(2,4$, 6 e $8 \mathrm{mg} \mathrm{L}^{-1}$ ), sendo que não se adicionou analito à primeira solução. Após a adição de iodo, o volume foi completado para $50 \mathrm{~mL}$ com CFA-C $10 \% \mathrm{v} \mathrm{v}^{-1}$ em $\mathrm{pH} 8$.

A exatidão do método foi avaliada por experimentos de adição e recuperação. Assim, para a realização do experimento, 4 amostras foram escolhidas, priorizando-se aquelas que não apresentaram resultados concordantes entre o método de adições de padrão e o método de compatibilização de matriz. Para isso, preparou-se uma solução a $4 \%(\mathrm{~m} / \mathrm{v})$ de amostra em meio de CFA-C $10 \% \mathrm{v} \mathrm{v}^{-1} \mathrm{em} \mathrm{pH} \mathrm{8,} \mathrm{retirou-}$ -se uma alíquota de $25 \mathrm{~mL}$ e transferiu-se para um balão volumétrico de $50 \mathrm{~mL}$. Nesses balões volumétricos foram adicionados volumes de solução padrão de iodo para que a concentração final fosse igual a $2 \mathrm{mg} \mathrm{L}^{-1}$, totalizando 3 replicatas para cada amostra. $\mathrm{O}$ branco foi considerado como a amostra diluída sem a adição do analito. Após a adição de iodo completou-se o volume com CFA-C $10 \% \mathrm{v} \mathrm{v}^{-1} \mathrm{em} \mathrm{pH} 8$.

\section{RESULTADOS E DISCUSSÃO}

\section{Avaliação do efeito de memória}

A ocorrência de efeitos de memória durante a aspiração de solução contendo iodeto em ICP OES com configuração axial foi avaliada pelo monitoramento das intensidades de emissão das linhas de iodo em função do tempo nos meios de CFA-C $10 \% \mathrm{v} \mathrm{v}^{-1} \mathrm{em} \mathrm{pH} 8$ e de $\mathrm{HNO}_{3}$ $1 \% \mathrm{v} \mathrm{v}^{-1}$. A Figura 1 apresenta o comportamento das duas linhas de emissão mais intensas de iodo (178,215 e 182,976 nm). Procurou-se manter o $\mathrm{pH}$ igual a 8 na solução de CFA-C para evitar a deterioração da tocha de quartzo, que comumente ocorre em $\mathrm{pH}$ acima de $9 . \mathrm{Na}$ Figura 1 nota-se que, no momento que a solução em meio de CFA-C é aspirada, há um pico na intensidade de iodo e esta se mantém estabilizada até o momento em que a aspiração da solução é cessada. Nesse momento, a intensidade diminui bruscamente até a linha de base. Por outro lado, quando a solução em meio de $\mathrm{HNO}_{3}$ é aspirada se observa ascensão gradual do sinal e lenta tendência para estabilização; ao cessar a aspiração da solução, o sinal diminui de forma lenta, sem retornar completamente até a linha de base. Esse comportamento é típico da ocorrência de efeito de memória, que pode ser atribuído à formação dos

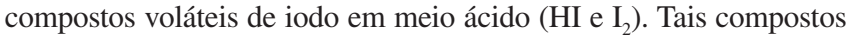
podem ser retidos na superfície da câmara de nebulização e tubulação de transporte, gerando instabilidades de sinais em medidas posteriores e, consequentemente, erros sucessivos nas medidas. ${ }^{22}$ Portanto, para evitar a formação dos compostos voláteis de iodo e, por consequência, o efeito de memória, devem ser utilizadas soluções alcalinas preparadas em meios contendo $\mathrm{NaOH}$, TMAH ou CFA-C nos sistemas de introdução de amostra e no preparo das mesmas. ${ }^{8}$

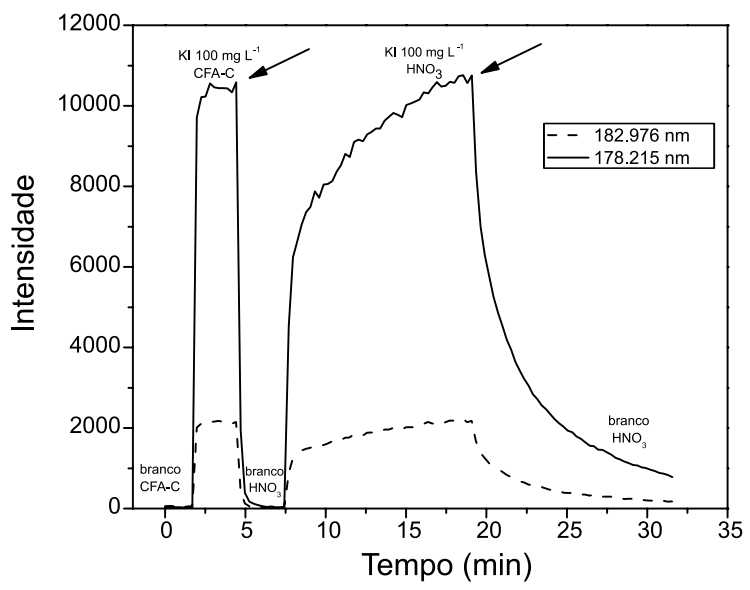

Figura 1. Intensidades de emissão de iodo em função do tempo nos meios de CFA-C $10 \% v v^{-1}$ e de $\mathrm{HNO}_{3} 1 \% v v^{-1}$ (a seta em cada ponto representa o instante no qual a aspiração da solução analítica foi interrompida)

O mecanismo de ação do CFA-C com o iodo ainda é desconhecido, porém alguns estudos demonstraram a eficiência desse reagente comparativamente a outros meios alcalinos. Gélinas et al. ${ }^{23}$ determinaram iodo em amostras nutricionais e biológicas por ICP-MS e usaram soluções de $\mathrm{NaOH}$ e de CFA-C para coletar os produtos de combustão. Os autores observaram menores limites de detecção e quantificação, efeito de memória nulo devido à estabilização de iodo dissolvido na fase líquida e maior exatidão ao utilizar CFA-C.

\section{Avaliação do desempenho dos ICP OESs com configuração axial ou radial}

Os resultados obtidos a partir dos planejamentos $2^{3}+$ Ponto Central + Estrela (Tabela 1S, material suplementar) indicaram que para a configuração axial as variáveis mais significativas foram a vazão do gás de nebulização (Gas) e a potência aplicada de radiofrequência (Pot). Já para a configuração radial, além das variáveis citadas anteriormente, a altura de observação também se mostrou como um parâmetro relevante. O intervalo estudado para a vazão de introdução da amostra não se mostrou importante para os experimentos realizados.

Ao aplicar um teste $t$ ao nível de confiança de $95 \%$ pode-se chegar a modelos matemáticos, referentes às configurações axial e radial e com esses modelos foram construídos os gráficos de contorno mostrados nas Figuras 2, 3 e 4. Os valores indicados no interior das figuras correspondem às intensidades de emissão da linha mais intensa de iodo, normalizadas pelo logaritmo na base 10. Esse artifício matemático foi efetuado, pois as respostas das variáveis apresentaram valores com magnitudes muito discrepantes (variando de, por exemplo, 0,01 
a 10000). Assim, utilizando o logaritmo na base 10 como resposta, foi possível obter um bom ajuste do modelo matemático.

No gráfico de contorno da Figura 2 observa-se que há uma região ótima de trabalho, com intensidade de emissão do iodo da ordem de 10000. Essa região abrange potência aplicada de radiofrequência de 1,0 a $1,3 \mathrm{~kW}$ e vazão do gás de nebulização de 0,6 a $0,8 \mathrm{~L} \mathrm{~min}^{-1}$.

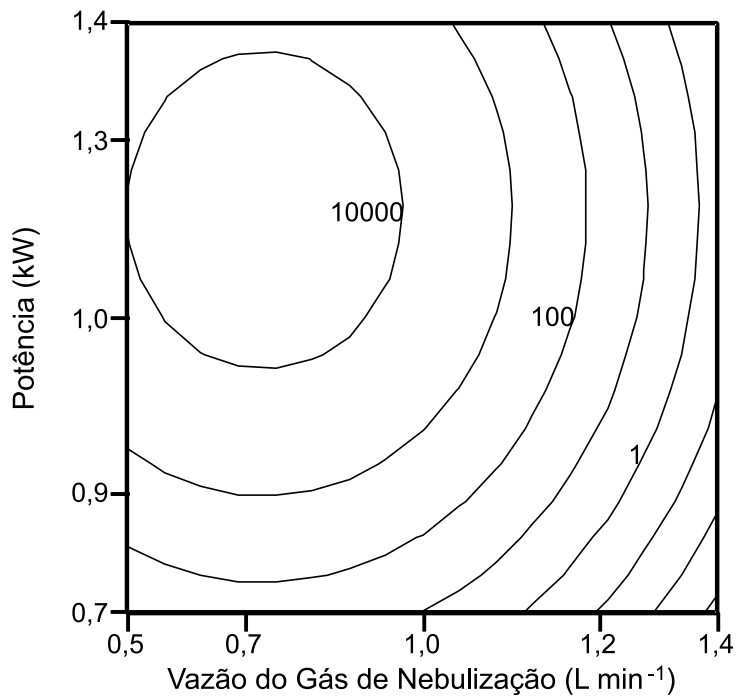

Figura 2. Gráfico de contorno obtido com planejamento fatorial em ICP OES com configuração axial. Variáveis: vazão do gás de nebulização versus potência aplicada

Nos gráficos de contorno das Figuras 3 e 4, observa-se que os maiores valores de intensidade de iodo foram obtidos quando foram utilizadas menor vazão do gás de nebulização, maior potência aplicada de radiofrequência e menor altura de observação. Assim, de acordo com valores reportados na literatura ${ }^{24,25}$ e com os experimentos efetuados, as condições de operação para máxima sensibilidade para o ICP OES com configuração axial foram obtidas em potência aplicada de $1,3 \mathrm{~kW}$, vazão do gás de nebulização de $0,8 \mathrm{~L} \mathrm{~min}^{-1}$ e vazão de introdução da amostra de $2,8 \mathrm{~mL} \mathrm{~min}^{-1}$ e para a configuração radial foram obtidas em potência aplicada de $1,3 \mathrm{~kW}$, vazão do gás de nebulização de $0,6 \mathrm{~L} \mathrm{~min}^{-1}$, vazão de introdução de amostra de $2,8 \mathrm{~mL}$ $\min ^{-1}$ e altura de observação de $8 \mathrm{~mm}$. Optou-se por utilizar a vazão

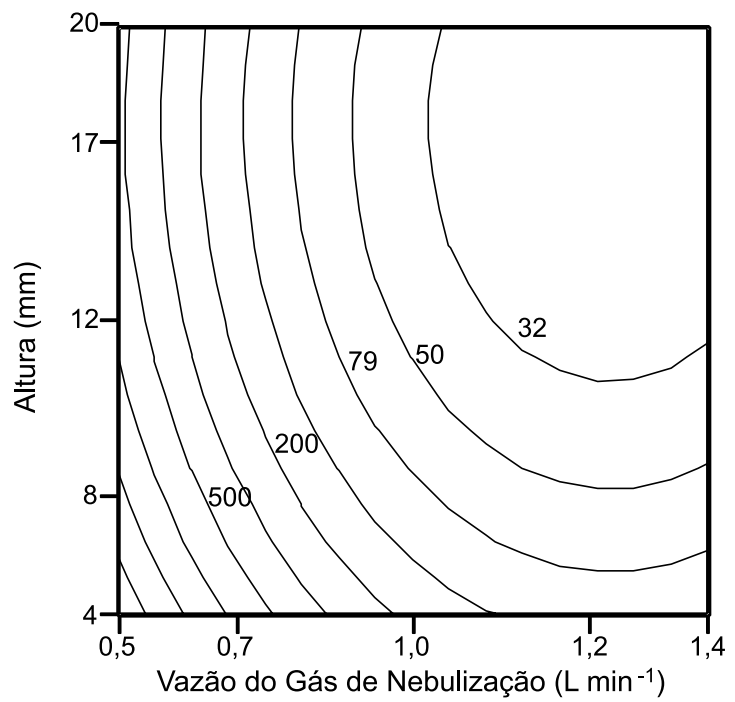

Figura 3. Gráfico de contorno obtido com planejamento fatorial em ICP OES com configuração radial. Variáveis: vazão do gás de nebulização versus altura de observação

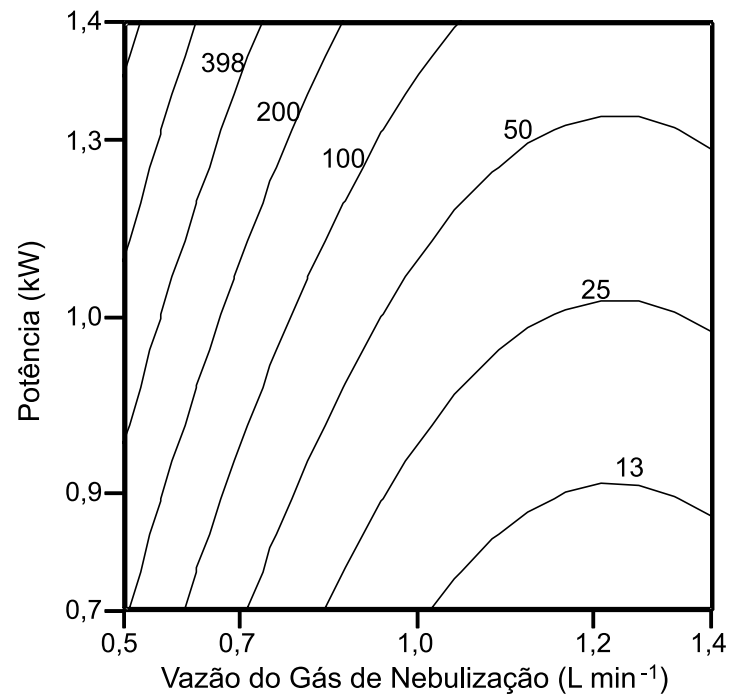

Figura 4. Gráfico de contorno obtido com planejamento fatorial em ICP OES com configuração radial. Variáveis: vazão do gás de nebulização versus potência aplicada

de introdução de amostra de $2,8 \mathrm{~mL} \mathrm{~min}^{-1}$ porque, apesar do intervalo estudado dessa variável não ser significativo, o planejamento indicava a aplicação de uma vazão maior.

Esse fato observado pode ser proveniente do uso de nebulizador com ranhura em V. Esse nebulizador é indicado para análise de suspensões porque é menos susceptível a entupimentos, porém sua geometria não é totalmente adequada para a geração do aerossol e, para superar essa ineficiência, recomenda-se maior vazão de introdução de amostra. ${ }^{21}$

Para avaliar as linhas de emissão de iodo é importante verificar suas intensidades relativas, porém esse não é o único parâmetro que determina qual é a linha mais recomendável para determinações analíticas.

A linha mais intensa encontra-se em 178,215 nm, sendo seguida pela linha em $182,976 \mathrm{~nm}$. Considerando-se esse aspecto e as medidas efetuadas dos brancos analíticos e da solução padrão contendo iodo $100 \mathrm{mg} \mathrm{L}^{-1}$ no meio de CFA-C, foram calculados os valores de SBR, BEC e LOD para as configurações axial e radial (Tabela $2 \mathrm{~S}$, material suplementar).

Ao avaliar os limites de detecção de cada configuração, verifica-se que aqueles obtidos no equipamento axial foram aproximadamente 5 vezes melhores que aqueles na configuração radial. Portanto, tal como esperado, a configuração axial mostrou-se mais sensível que a radial e pode ser indicada para a determinação de iodo, principalmente em amostras com menores concentrações desse elemento.

Além do menor limite de detecção, nota-se também que a linha mais intensa $(178,215 \mathrm{~nm})$ apresentou maior valor de SBR do que a menos intensa $(184,382 \mathrm{~nm})$ para ambas as configurações. Quanto maior o valor de SBR, menor a contribuição do sinal de fundo para a análise.

Menores LOD's foram estabelecidos para as duas linhas mais intensas, ou seja, 178,215 e 182,976 nm. Na configuração axial, foram obtidos valores próximos para a segunda e terceira linhas mais intensas $(182,976$ e 179,847 nm). Apesar de suas intensidades relativas serem diferentes, os desvios padrão relativos utilizados para o cálculo do limite de detecção foram de 11,05 e 5,28\%, respectivamente, para as linhas 182,976 e $179,847 \mathrm{~nm}$.

Ainda para comparar o desempenho das linhas para o iodo, foram obtidas curvas analíticas de calibração para cada um dos comprimentos de onda na configuração axial. Os parâmetros das curvas de calibração (inclinação e intercepto) foram utilizados para 
calcular as concentrações previstas de iodo. Essas concentrações previstas foram comparadas com as concentrações reais por meio do cálculo do RMSEP (raiz quadrada da soma dos quadrados dos erros de previsão), sendo esse um parâmetro que avalia o ajuste da curva de calibração (Equação 1). ${ }^{26}$

$$
\sqrt{\frac{\sum\left(y_{i}-y_{i i}\right)^{2}}{n}}
$$

onde: $\mathrm{y}_{\mathrm{i}}$ e $\mathrm{y}_{\mathrm{ii}}$ são os valores reais e previstos, respectivamente, e $n$ é o número de amostra do conjunto de calibração.

Os valores de RMSEP obtidos para as linhas de emissão de iodo encontram-se na Tabela 3S, material suplementar.

O valor de RMSEP obtido para a linha $178,215 \mathrm{~nm}$ foi de $0,0943 \mathrm{mg} \mathrm{L}^{-1}$, indicando que concentrações inferiores a esse valor provavelmente serão difíceis de serem determinadas. Para a linha de emissão menos intensa, ou seja, 184,382 nm, o valor de RMSEP foi $0,407 \mathrm{mg} \mathrm{L}^{-1}$.

A faixa linear de calibração encontrada para a linha de emissão $178,215 \mathrm{~nm}$ na configuração axial correspondeu à região de concentração entre 0,3 a $600 \mathrm{mg} \mathrm{L}^{-1}$, apresentando coeficiente de correlação $\left(\mathrm{R}^{2}\right)$ igual a 0,991. Já para a configuração radial, a faixa linear de calibração foi de 1,8 a $1000 \mathrm{mg} \mathrm{L}^{-1}$, apresentando $\mathrm{R}^{2}$ igual a 0,999 .

\section{Avaliação de interferentes}

Os cátions $\mathrm{Na}^{+}$e $\mathrm{K}^{+}$foram avaliados como possíveis interferentes, pois as amostras de sal investigadas apresentavam teores elevados de $\mathrm{Na}^{+}$e a adição de iodo ao sal de cozinha pode ser feita por meio de KI ou $\mathrm{KIO}_{3}$. Outra amostra investigada foi o sal conhecido como light. Esse sal é especialmente interessante, pois é rico em $\mathrm{K}^{+}$totalizando até $50 \%$ da composição.

As Figuras 5 e $1 \mathrm{~S}$ a $4 \mathrm{~S}$ (material suplementar) representam os efeitos causados por $\mathrm{Na}^{+}, \mathrm{K}^{+}$e da mistura $\mathrm{Na}^{+}$e $\mathrm{K}^{+}$, na intensidade das cinco linhas de iodo $(178,215 ; 182,976 ; 179,847 ; 184,382$ e $206,163 \mathrm{~nm}$ ). A linha tracejada é a média normalizada em 100 da intensidade de iodo na ausência de concomitantes e as linhas contínuas correspondem à média normalizada da intensidade de iodo $\pm 10 \%$.

Nas Figuras 5 e $1 \mathrm{~S}$ a $4 \mathrm{~S}$ (material suplementar) pode-se observar a ocorrência de interferência positiva sobre iodo, causada por $\mathrm{Na}^{+} \mathrm{e}$ $\mathrm{K}^{+}$. No entanto, a interferência de $\mathrm{Na}^{+}$começa a ser pronunciada a partir de $10000 \mathrm{mg} \mathrm{L}^{-1}$, a de $\mathrm{K}^{+}$e da mistura $\mathrm{Na}^{+}$e $\mathrm{K}^{+}$se manifestaram a partir de $2000 \mathrm{mg} \mathrm{L}^{-1}$, o que demonstra que a interferência de $\mathrm{K}^{+}$ foi a mais pronunciada.

Ao analisar o comportamento das linhas de emissão para as situações em que os cátions $\mathrm{Na}^{+}$e/ou $\mathrm{K}^{+}$estavam presentes, verifica-se o mesmo perfil para todas as linhas, exceto para a linha $182,976 \mathrm{~nm}$ (Figura 1S), que apresentou interferência mínima (aproximadamente $8 \%$ ) de $\mathrm{Na}^{+}$para o intervalo estudado de concentrações.

Esse tipo de interferência pode estar associado a efeitos relacionados às etapas de nebulização, transporte e efeitos energéticos no plasma. Ácidos utilizados no preparo da amostra e elementos facilmente ionizáveis são frequentemente os responsáveis pelos efeitos de matriz em ICP OES. ${ }^{21,27}$

Elementos facilmente ionizáveis geralmente se encontram em concentrações elevadas em vários tipos de amostras e no caso do sal de cozinha o $\mathrm{Na}^{+}$é o elemento majoritário.

A fim de contornar essas interferências não espectrais algumas alternativas podem ser adotadas, tal como, a compatibilização de matriz, que consiste na utilização de soluções analíticas de calibração preparadas em meio semelhante ao meio no qual o analito se encontra. Caso essa estratégia não seja eficiente, o método das adições de padrão pode ser utilizado.
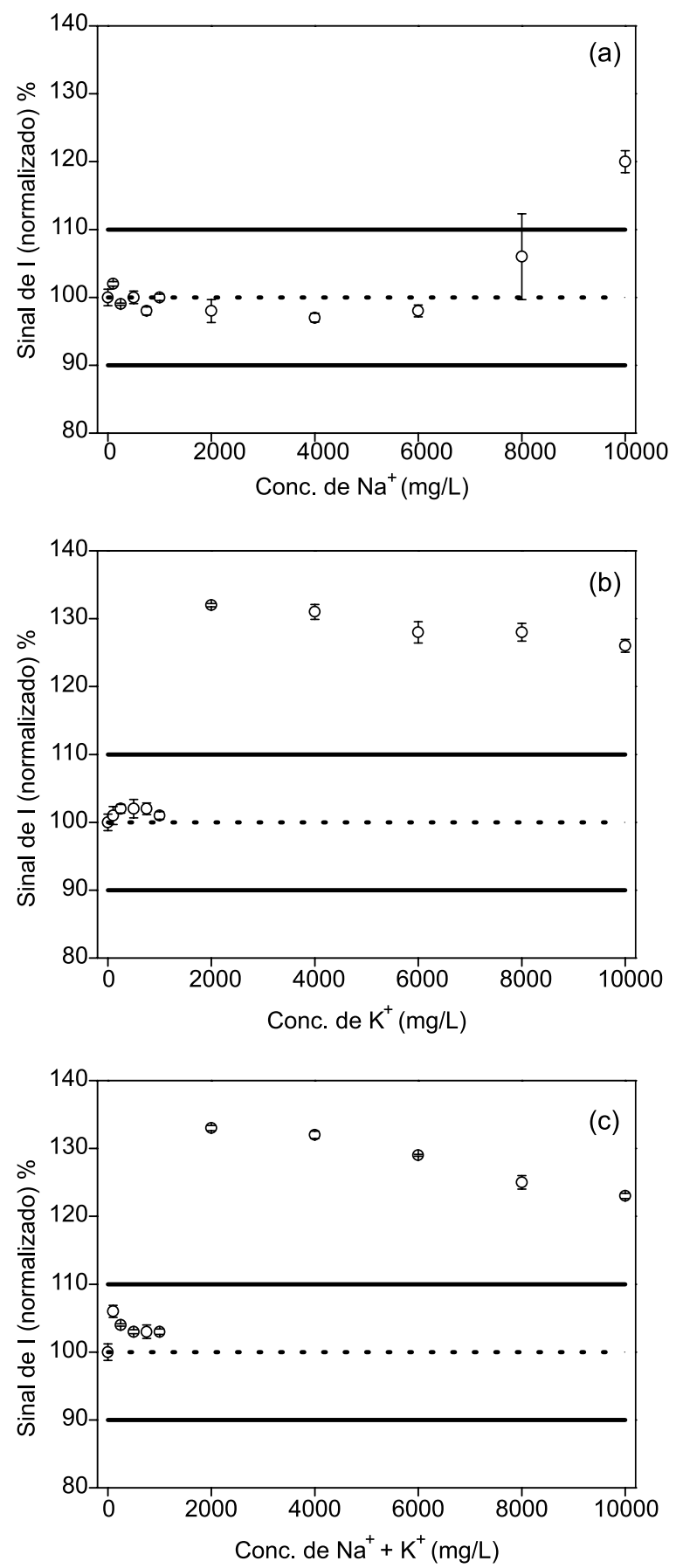

Figura 5. Efeitos da presença de interferentes na linha de emissão de iodo 178,215 nm: (a) $\mathrm{Na}^{+}$, (b) $\mathrm{K}^{+}$e de (c) $\mathrm{Na}^{+}+\mathrm{K}^{+}(1: 1)$

\section{Determinação de iodo em sal de cozinha}

Frente à importância nutricional e ao quadro mundial de deficiência de iodo, cada vez mais se faz necessário o aperfeiçoamento de técnicas analíticas para a determinação desse elemento em alimentos, principalmente em sal de cozinha por ser a principal fonte de iodo para a população. O teor de iodo adicionado ao sal de cozinha é baixo em relação à concentração de $\mathrm{Na}^{+}$. Para atingir a concentração mínima de iodo quantificável ( $\mathrm{LOQ}=0,3 \mathrm{mg} \mathrm{L}^{-1}$ ) em ICP OES com configuração axial, o teor de $\mathrm{Na}^{+}$na solução é elevado (8000 $\left.\mathrm{mg} \mathrm{L}^{-1}\right)$.

Sistemas axiais apresentam limitações na análise de amostras com elevados teores de sólidos dissolvidos, devido à deposição de sais no tubo central da tocha e consequente entupimento e diminuição de sua 
vida útil. O desgaste da tocha de quartzo ocorre porque o $\mathrm{Na}^{+}$acelera a mudança de fase do quartzo, tornando-o quebradiço. Assim, para prolongar a vida útil da tocha de quartzo aumentou-se a vazão do gás auxiliar para 2,25 $\mathrm{L} \mathrm{min}^{-1}$, desabilitou-se no programa de controle do instrumento a aceleração da bomba peristáltica e foi utilizada solução de limpeza de CFA-C $10 \% \mathrm{v} \mathrm{v}^{-1}$ durante $1 \mathrm{~min}$, entre as leituras. ${ }^{28}$

Na determinação de iodo nas amostras de sal de cozinha foi adotada a estratégia de compatibilização de matriz, devido à interferência posi-

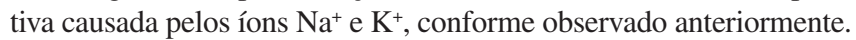
A linha de iodo utilizada foi a I 178,215 nm. Visando corrigir o efeito de matriz observado mesmo quando se utilizou compatibilização de matriz, optou-se por quantificar iodo nas amostras pelo método de calibração por adições de padrão. Deve-se ressaltar que a compatibilização de matriz implica em uma maior similaridade entre os meios das soluções de calibração e das amostras, entretanto essa similaridade pode ainda não implicar em comportamento idêntico, particularmente quando se trabalha com amostras complexas. Os teores médios de iodo determinados nas 9 amostras de sal de cozinha por compatibilização de matriz e pelo método das adições de padrão encontram-se na Tabela 2.

Tabela 2. Teores médios e desvios padrão de iodo, obtidos por ICP OES com configuração axial em amostras de sal de cozinha utilizando compatibilização de matriz $(\mathrm{n}=3)$ e método das adições de padrão $(\mathrm{n}=3)^{*}$

\begin{tabular}{lcc}
\hline \multirow{2}{*}{ Amostra } & \multicolumn{2}{c}{ Iodo $\left(\mathrm{mg} \mathrm{kg}^{-1}\right)$} \\
\cline { 2 - 3 } & $\begin{array}{c}\text { Compatibilização de } \\
\text { matriz }\end{array}$ & $\begin{array}{c}\text { Método das adições de } \\
\text { padrão }\end{array}$ \\
\hline Sal comum 1 & $19,82 \pm 4,91$ & $21,80 \pm 5,52$ \\
Sal comum 2 & $17,29 \pm 2,74$ & $17,79 \pm 3,55$ \\
Sal comum 3 & $32,50 \pm 4,55$ & $25,91 \pm 1,99$ \\
Sal light 1 & $30,89 \pm 6,23$ & $28,20 \pm 3,61$ \\
Sal light 2 & $19,67 \pm 2,57$ & $21,10 \pm 1,75$ \\
Sal marinho 1 & $34,72 \pm 3,71$ & $12,36 \pm 2,65$ \\
Sal marinho 2 & $14,33 \pm 2,61$ & $15,65 \pm 2,37$ \\
Sal marinho 3 & $26,33 \pm 3,80$ & $19,06 \pm 2,91$ \\
Sal marinho 4 & $13,15 \pm 1,56$ & $14,72 \pm 2,74$ \\
\hline
\end{tabular}

*O desvio nesse caso foi calculado para medidas sucessivas da mesma solução e, portanto, reflete o desempenho instrumental.

As denominações sal comum 1, 2 e 3 e sucessivamente foram usadas para diferenciar as marcas analisadas pertencentes ao mesmo tipo de sal.

Na Tabela 2 nota-se que para as duas estratégias de quantificação adotadas, a maioria das amostras apresentou teores de iodo acima do valor mínimo exigido pela ANVISA $\left(20 \mathrm{mg} \mathrm{kg}^{-1}\right)$. Algumas amostras apresentaram valores abaixo da legislação, dentre elas as mais discrepantes foram duas marcas de sal marinho e, outra relativamente baixa, uma marca de sal comum. Na quantificação por compatibilização de matriz o LOD encontrado foi de $3,55 \mathrm{mg} \mathrm{kg}^{-1}$.

Em ambos os métodos, algumas amostras apresentaram valores altos de desvios padrão relativos. Esse fato pode ser causado pelo alto teor de $\mathrm{Na}^{+}$encontrado na matriz, que dificulta os processos de nebulização e transporte e também pela alta energia de ionização do iodo quando comparada ao sódio.

Ao comparar os resultados encontrados nos dois métodos (compatibilização de matriz e adições de padrão) utilizados para a quantificação de iodo nas amostras de sal de cozinha, nota-se que os teores médios para a maioria das amostras ficaram próximos, exceto para as amostras de sais marinhos 1 e 3 e sal comum 3, que apresentaram diferenças significativas. Esse aspecto provavelmente decorre do fato que a compatibilização de matriz não foi efetuada de maneira totalmente eficaz, já que o sal marinho é composto por outros sais além do $\mathrm{NaCl}$. Para comprovar a concordância entre os valores obtidos pelos métodos de compatibilização de matriz e adições de padrão, foi calculada uma equação de primeiro grau que descreve a concentração de iodo obtida em ambas as estratégias. ${ }^{29}$ As amostras que apresentaram resultados claramente discrepantes não foram incluídas nos cálculos.

A equação obtida apresentou um $\mathrm{R}^{2}$ igual a 0,9664 e quanto mais próximo de 1, mais semelhantes são os valores das duas estratégias de calibração. Assim, os métodos apresentaram boa correlação e pode-se dizer que há uma boa concordância entre os resultados.

A exatidão do método foi avaliada por experimentos de adição e recuperação, empregando-se as amostras que não apresentaram resultados concordantes entre os métodos das adições de padrão e de compatibilização de matriz. Assim, as amostras estudadas foram sal comum 3, sal light 1, sais marinhos 1 e 3 . As porcentagens de recuperação obtidas foram 104,9; 114,1; 106,7 e 112,6\% para o sal comum 3, sal light 1, sal marinho 1 e sal marinho 3, respectivamente.

As recuperações estão acima de $100 \%$, o que indica a possibilidade de ocorrência de erros sistemáticos positivos. Pode-se afirmar que o método de compatibilização de matriz apresentou boa exatidão e confiabilidade, já que os valores de recuperação encontraram-se na margem de $120 \%$ de tolerância. Considerando-se os baixos teores de iodo, adotou-se a faixa de tolerância de 80 a $120 \%$.

\section{CONCLUSÃO}

São poucos os estudos que enfocam a determinação de iodo por ICP OES com configuração axial. Assim, torná-lo viável para essa aplicação aumenta sua capacidade analítica, além de ser mais uma alternativa para a determinação de iodo.

O iodo pode ser determinado por ICP OES com configuração axial utilizando as condições operacionais otimizadas, isto é, vazão do gás de nebulização de $0,8 \mathrm{~L} \mathrm{~min}^{-1}$, potência de radiofrequência de $1,3 \mathrm{~kW}$ e vazão de introdução de amostra de $2,8 \mathrm{~mL} \mathrm{~min}^{-1}$. Salienta-se que esses valores são para o sistema de introdução de amostra composto por câmara Sturman-Masters e nebulizador com ranhura em $\mathrm{V}$, indicados para a análise de sal de cozinha devido ao alto teor de sólidos dissolvidos das soluções que são introduzidas no equipamento. Ressalta-se que a configuração axial mostrou ser mais sensível que a configuração radial mediante aos valores de LOD's obtidos, que foram na ordem de 5 vezes menores.

O meio contendo CFA-C mostrou-se mais eficiente do que $\mathrm{HNO}_{3}$ para evitar efeito de memória e foi adotado para a execução de todo o trabalho analítico.

A linha de emissão de iodo mais sensível, I 178,215 nm, apresentou baixo $\operatorname{LOD}\left(0,10 \mathrm{mg} \mathrm{L}^{-1}\right)$ e alto valor de SBR. Entretanto, essa linha foi afetada por interferências causadas por $\mathrm{Na}^{+}$e $\mathrm{K}^{+}$. A segunda linha mais intensa (I 182,976 nm) apresentou LOD igual a $0,68 \mathrm{mg} \mathrm{L}^{-1}$, porém baixa interferência causada por $\mathrm{Na}^{+}$em concentração de até $10000 \mathrm{mg} \mathrm{L}^{-1}$.

A utilização de soluções analíticas de calibração contendo $\mathrm{Na}^{+}$ mostrou-se uma boa alternativa para corrigir a interferência desse cátion nos sinais de iodo. Os valores foram similares, para a maioria das amostras, quando se comparou a compatibilização de matriz com o método das adições de padrão. O método de compatibilização de matriz apresentou boa exatidão e confiabilidade nos experimentos de adição e recuperação, obtendo-se valores de recuperação de até $120 \%$.

\section{MATERIAL SUPLEMENTAR}

Disponível em http://quimicanova.sbq.org.br, em arquivo pdf, com acesso livre. 


\section{AGRADECIMENTOS}

Ao Conselho Nacional de Desenvolvimento Científico e Tecnológico (CNPq), à Coordenação de Aperfeiçoamento de Pessoal de Nível Superior (CAPES) e à Fundação de Amparo à Pesquisa do Estado de São Paulo (FAPESP, Processo 2006/59083-9) pelo apoio.

\section{REFERÊNCIAS}

1. Benoist, B.; Andersson, A.; Egli, I.; Takkouche, B.; Allen, H.; Iodine Status worldwide: WHO Global Database on Iodine Deficiency, Department of Nutrition for Health and Development World Health Organization: Geneva, 2004.

2. Cozzolino, S. M. F.; Biodisponibilidade de nutrientes, $1^{\mathrm{a}}$ ed., Manole: Barueri, 2005.

3. Ministério da Saúde, Resolução ANVISA RDC n 32, 25/2/2003.

4. Marczenko, Z.; Separations and Spectrophotometric Determination of Elements, Ellis Horwood: Chichester, 1986.

5. Knapp, G.; Maichin, B.; Fecher, P.; Hasse, S.; Fresenius J. Anal. Chem. 1998, 362, 508.

6. Amount, G.; Tressol, J. C.; Analyst 1986, 111, 841.

7. Bermejo-Barrera, P.; Aboal-Somoza, M.; Bermejo-Barrera, A.; J. Anal. At. Spectrom. 1999, 14, 1009.

8. Tagami, K.; Uchida, S.; Hirai, I.; Tsukada, H.; Takeda, H.; Anal. Chim. Acta 2006, 570, 88.

9. Varga, I.; Microchem. J. 2007, 85, 127.

10. Gouveia, S. T.; Silva, F. V.; Costa, L. M.; Nogueira, A. R. A.; Nóbrega, J. A.; Anal. Chim. Acta 2001, 445, 269.

11. Houseaux, J.; Mermet, J. M.; J. Anal. At. Spectrom. 2000, 15, 979.

12. Krengel-Rothensee, K.; Richter, U.; Heitland, P.; J. Anal. At. Spectrom. 1999, 14, 699 .
13. Schulz, O.; Heitland, P.; Fresenius J. Anal. Chem. 2001, 371, 1070.

14. Naozuka, J.; Veiga, M. A. M. S.; Oliveira, P. V.; Oliveira, E.; J. Anal. At. Spectrom. 2003, 18, 917.

15. Montaser, A., ed.; Inductively Coupled Plasma Mass Spectrometry, Wiley: New York, 1998.

16. Nóbrega, J. A.; Santos, M. C.; Sousa, R. A.; Cadore, S.; Spectrochim. Acta, Part B 2005, 61, 465 .

17. Barros Neto, B.; Scarminio, I. S.; Bruns, R. E.; Como fazer experimentos, Ed. da UNICAMP: Campinas, 2003.

18. Manual de controle do ICP OES com configuração axial (ICP Expert versão 1.2); Varian, Mulgrave, Victoria, Austrália, 1997.

19. Thomsen, V.; Roberts, G.; Burgess, K.; Spectroscopy 2000, 15, 33.

20. Montaser, A.; Golightly, D. W.; Inductively Coupled Plasmas in Analytical Atomic Spectroscopy, VCH Publisher: New York, 1992.

21. Trevizan, L. C.; Tese de Doutorado, Universidade Federal de São Carlos, Brasil, 2007.

22. Al-Ammar, A.; Reitznerová, E.; Barnes, R. M.; Fresenius J. Anal. Chem. 2001, 370, 479

23. Gélinas, Y.; Krushevska, A.; Barnes, R. M.; Anal. Chem. 1998, 70, 1021.

24. Silva, F. V.; Trevizan, L. C.; Silva, C. S.; Nogueira, A. R. A.; Nóbrega, J. A.; Spectrochim. Acta, Part B 2002, 57, 1905.

25. Trevizan, L. C.; Vieira, E. C.; Nogueira, A. R. A.; Nóbrega, J. A.; Spectrochim. Acta, Part B 2005, 60, 575 .

26. Sharaf, M. A.; Illman, D. L.; Kowalski, B. R.; Chemometrics, John Wiley \& Sons: New York, 1986.

27. Giné-Rosias, M. F.; Espectrometria de Emissão Atômica com Plasma Acoplado Indutivamente (ICP-AES), CENA: Piracicaba, 1998.

28. Matias, T. B.; Tese de Doutorado, Universidade Federal de São Carlos, Brasil, 2007.

29. Miller, J. C.; Miller, J. N.; Estadística para Química Analítica, AddisonWesley Iberoamericana: EUA, 1993. 


\section{AVALIAÇÃO DE ICP OES COM CONFIGURAÇÃO AXIAL OU RADIAL PARA DETERMINAÇÃO DE IODO EM SAL DE COZINHA}

Adriana A. Oliveira\#, Joaquim A. Nóbrega* e Edenir R. Pereira-Filho

Departamento de Química, Universidade Federal de São Carlos, CP 676, 13560-970 São Carlos - SP, Brasil

Lilian C. Trevizan"\#

Centro de Energia Nuclear na Agricultura, Universidade de São Paulo, CP 96, 13400-970 Piracicaba - SP, Brasil
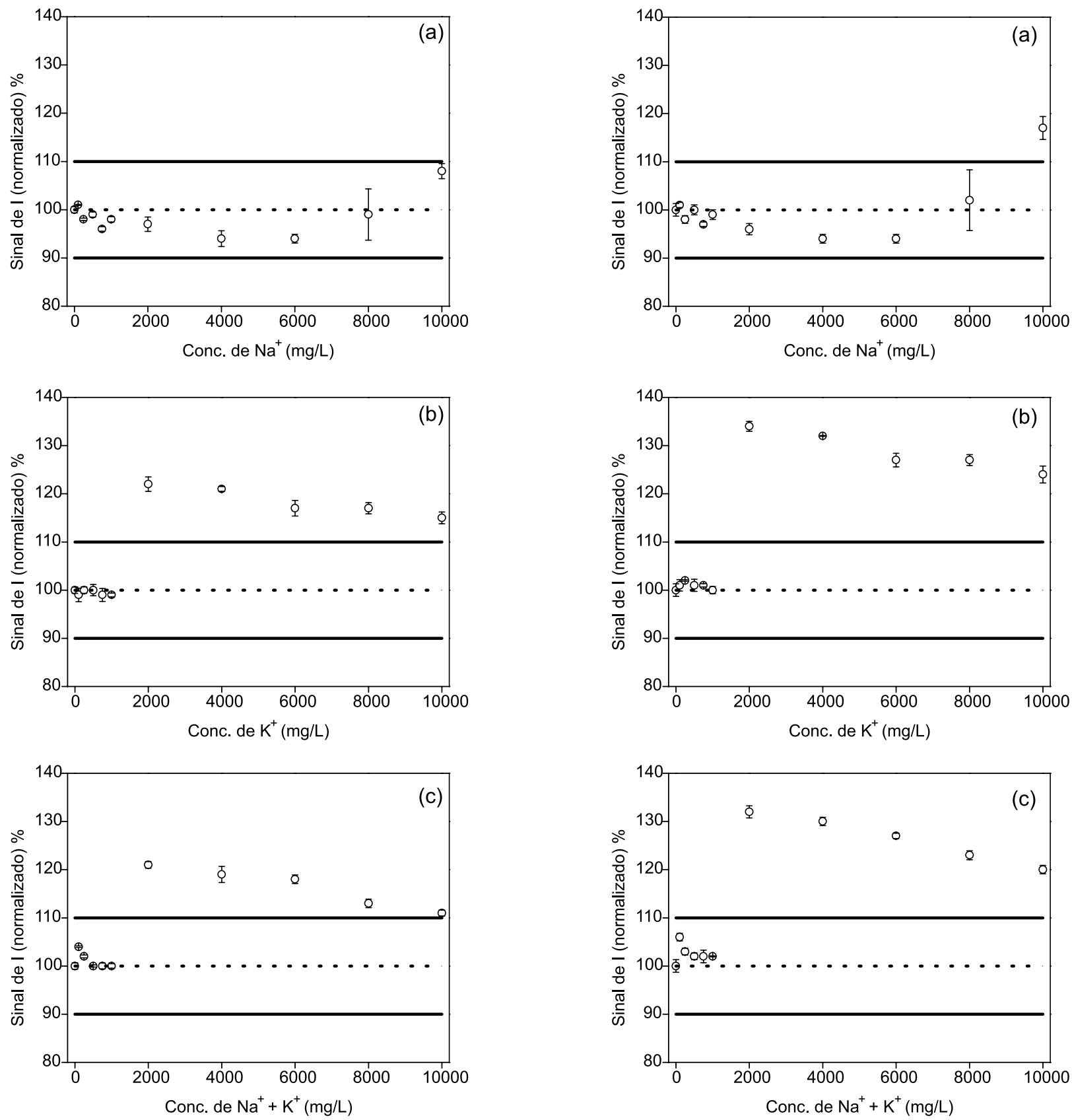

Figura 1S. Efeitos da presença de interferentes na linha de emissão de iodo 182,976 nm: (a) $\mathrm{Na}^{+}$, (b) $\mathrm{K}^{+}$e de (c) $\mathrm{Na}^{+}+\mathrm{K}^{+}(1: 1)$

Figura 2S. Efeitos da presença de interferentes na linha de emissão de iodo 179,847 $\mathrm{nm}:$ (a) $\mathrm{Na}^{+}$, (b) $\mathrm{K}^{+}$e de (c) $\mathrm{Na}^{+}+\mathrm{K}^{+}(1: 1)$

*e-mail: djan@ufscar.br

"Endereço atual: Corn Products, Mogi Guaçu - SP

"\#Endereço atual: Divisão de Controle Sanitário e Ambiental, Unidade de Negócio do Médio Tietê, Cia. de Saneamento Básico do Estado de São Paulo, Botucatu - SP 

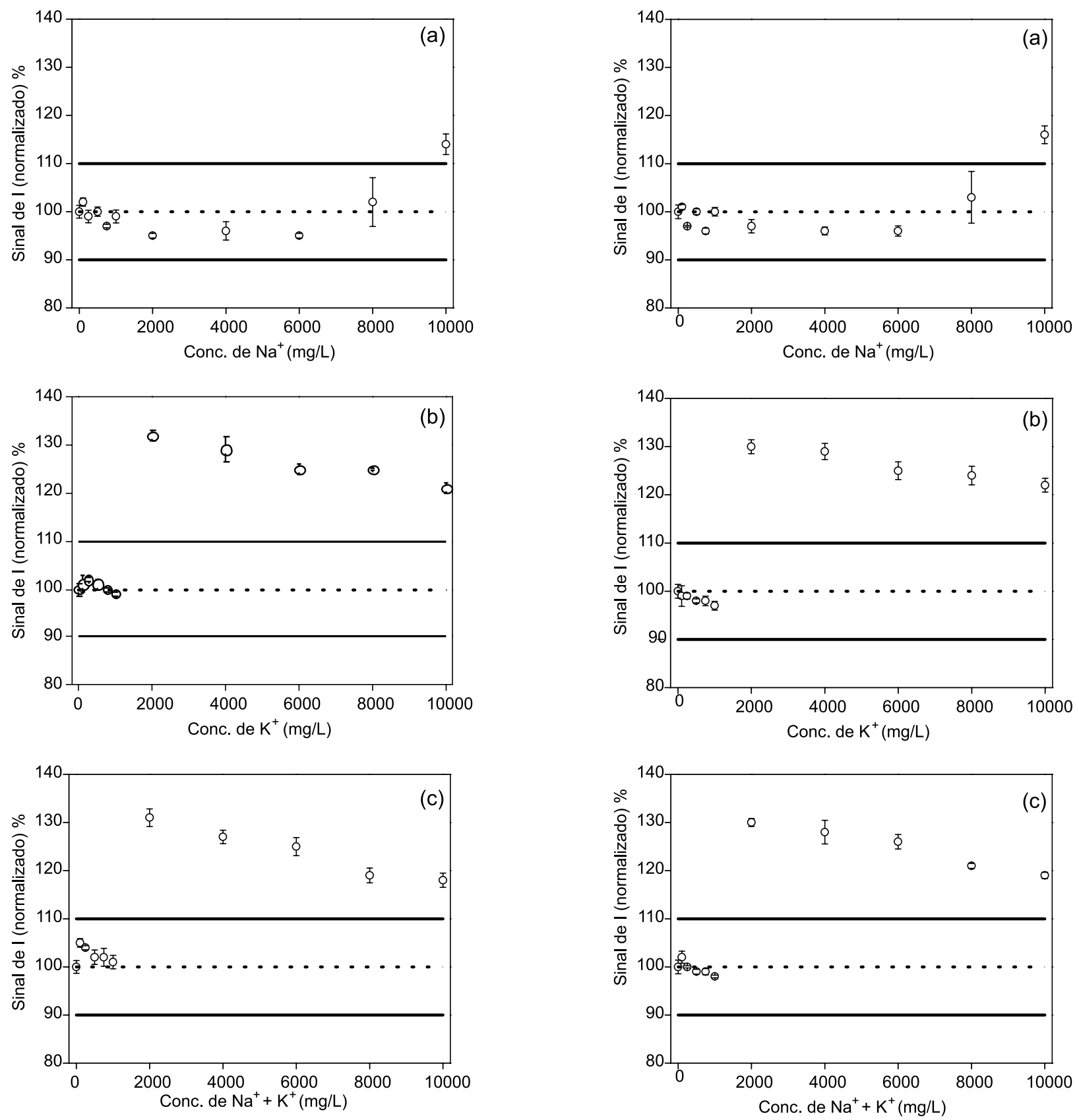

Figura 3S. Efeitos da presença de interferentes na linha de emissão de iodo 184,382 $\mathrm{nm}:$ (a) $\mathrm{Na}^{+}$, (b) $\mathrm{K}^{+}$e de (c) $\mathrm{Na}^{+}+\mathrm{K}^{+}(1: 1)$

Figura 4S. Efeitos da presença de interferentes na linha de emissão de iodo 206,163 nm: (a) $\mathrm{Na}^{+}$, (b) $\mathrm{K}^{+}$e de (c) $\mathrm{Na}^{+}+\mathrm{K}^{+}$(1:1) 
Tabela 1S. Planejamento fatorial efetuado para avaliação das condições operacionais do ICP OES axial e radial

\begin{tabular}{|c|c|c|c|c|c|c|c|c|}
\hline \multirow[t]{2}{*}{ Experimento } & \multicolumn{2}{|c|}{$\begin{array}{l}\text { Vazão do gás de nebulização } \\
\text { (1) }\end{array}$} & \multicolumn{2}{|c|}{$\begin{array}{l}\text { Potência de rádio frequência } \\
\qquad(2)\end{array}$} & \multicolumn{2}{|c|}{$\begin{array}{l}\text { Vazão de introdução de amostra / } \\
\text { Altura de observação (3) }\end{array}$} & \multicolumn{2}{|c|}{$\begin{array}{l}\text { Sinais obtidos } \\
\text { (média } \pm \text { D.P.) }\end{array}$} \\
\hline & Normalizado & Real & Normalizado & Real & Normalizado & Real & Axial & Radial \\
\hline 1 & -1 & 0,7 & -1 & 0,9 & -1 & $1,4 / 8$ & $3829 \pm 14,49$ & $199 \pm 4,31$ \\
\hline 2 & +1 & 1,2 & -1 & 0,9 & -1 & $1,4 / 8$ & 0 & $52,4 \pm 4,04$ \\
\hline 3 & -1 & 0,7 & +1 & 1,3 & -1 & $1,4 / 8$ & $11978 \pm 112,47$ & $640 \pm 4,77$ \\
\hline 4 & +1 & 1,2 & +1 & 1,3 & -1 & $1,4 / 8$ & 0 & $117 \pm 6,44$ \\
\hline 5 & -1 & 0,7 & -1 & 0,9 & +1 & $3,6 / 17$ & $2971 \pm 36,85$ & $57,0 \pm 4,56$ \\
\hline 6 & +1 & 1,2 & -1 & 0,9 & +1 & $3,6 / 17$ & 0 & $11,5 \pm 2,53$ \\
\hline 7 & -1 & 0,7 & +1 & 1,3 & +1 & $3,6 / 17$ & $14279 \pm 145,72$ & $418 \pm 5,69$ \\
\hline 8 & +1 & 1,2 & +1 & 1,3 & +1 & $3,6 / 17$ & 0 & $36,5 \pm 2,10$ \\
\hline 9 & 0 & 1,0 & 0 & 1,0 & 0 & $2,8 / 12$ & $3948 \pm 17,25$ & $44,3 \pm 1,36$ \\
\hline 10 & 0 & 1,0 & 0 & 1,0 & 0 & $2,8 / 12$ & $3755 \pm 17,88$ & $42,2 \pm 2,93$ \\
\hline 11 & 0 & 1,0 & 0 & 1,0 & 0 & $2,8 / 12$ & $3246 \pm 41,84$ & $28,2 \pm 1,74$ \\
\hline 12 & $-1,68$ & 0,5 & 0 & 1,0 & 0 & $2,8 / 12$ & $953 \pm 12,52$ & $441 \pm 5,35$ \\
\hline 13 & $+1,68$ & 1,4 & 0 & 1,0 & 0 & $2,8 / 12$ & 0 & $22,8 \pm 1,24$ \\
\hline 14 & 0 & 1,0 & $-1,68$ & 0,7 & 0 & $2,8 / 12$ & 0 & $8,37 \pm 4,11$ \\
\hline 15 & 0 & 1,0 & $+1,68$ & 1,4 & 0 & $2,8 / 12$ & $8901 \pm 26,59$ & $182 \pm 3,47$ \\
\hline 16 & 0 & 1,0 & 0 & 1,0 & $-1,68$ & $0,5 / 4$ & $2543 \pm 55,27$ & $135 \pm 3,94$ \\
\hline 17 & 0 & 1,0 & 0 & 1,0 & $+1,68$ & $4,5 / 20$ & $3910 \pm 45,71$ & $21,94,65$ \\
\hline
\end{tabular}

Tabela 2S. Valores de SBR, BEC e LOD para as linhas de emissão de iodo para configurações axial e radial

\begin{tabular}{|c|c|c|c|c|c|c|}
\hline \multirow{2}{*}{$\begin{array}{l}\text { Comprimento de } \\
\text { onda }(\mathrm{nm})\end{array}$} & \multicolumn{3}{|c|}{ Axial } & \multicolumn{3}{|c|}{ Radial } \\
\hline & SBR & $\mathrm{BEC}\left(\mathrm{mg} \mathrm{L}^{-1}\right)$ & $\operatorname{LOD}\left(\mathrm{mg} \mathrm{L}^{-1}\right)$ & SBR & $\mathrm{BEC}\left(\mathrm{mg} \mathrm{L}^{-1}\right)$ & $\operatorname{LOD}\left(\mathrm{mg} \mathrm{L}^{-1}\right)$ \\
\hline $\mathrm{I}^{*} 178,215$ & 311,2 & 0,32 & 0,10 & 40,2 & 2,49 & 0,62 \\
\hline I 179,847 & 23,5 & 4,25 & 0,67 & 10,6 & 9,42 & 4,23 \\
\hline I 182,976 & 48,5 & 2,06 & 0,68 & 9,77 & 10,2 & 3,24 \\
\hline I 184,382 & 8,38 & 11,9 & 1,56 & 1,92 & 52,1 & 14,1 \\
\hline I 206,163 & 9,09 & 11,0 & 1,17 & 2,27 & 44,0 & 8,31 \\
\hline
\end{tabular}

*I Linha atômica

Tabela 3S. Valores de RMSEP para as linhas de emissão de iodo

\begin{tabular}{cc}
\hline Comprimento de onda $(\mathrm{nm})$ & $\operatorname{RMSEP}\left(\mathrm{mg} \mathrm{L}^{-1}\right)$ \\
\hline $\mathrm{I}^{*} 178,215$ & 0,0943 \\
I 179,847 & 0,254 \\
I 182,976 & 0,238 \\
I 184,382 & 0,407 \\
I 206,163 & 0,460 \\
\hline
\end{tabular}

*I Linha atômica 